\title{
COMPACTNESS AND SEMI-CONTINUOUS CARRIERS
}

\author{
JACK G. CEDER
}

A real-valued function $f$ on a topological space $X$ is defined to be upper (lower) semi-continuous if the set $\{x: f(x) \geqq \lambda\}$ (resp. $\{x: f(x) \leqq \lambda\})$ is closed in $X$ for each real number $\lambda[3$, p. 101]. This notion has been generalized to a function from a topological space into some set of subsets of another topological space (cf. Hahn [2, p. 148] or Michael [4, p. 179]). More precisely, letting $a$ be some collection of nonempty subsets of $Y$, we say that a function $\Phi$ from $X$ to $Q$ is an upper (lower) semi-continuous carrier from $X$ to $Q$ if the set $\{x: \Phi(x) \subset U\}$ (resp. $\{x: \Phi(x) \cap U \neq \Lambda\}$ ) is open in $X$ for each open set $U$ in $Y$. Note that if $f$ is an u.s.c. (l.s.c.) real-valued function, then $\Phi$, defined by $\Phi(x)=\{r: r \leqq f(x)\}$, becomes an u.s.c. (l.s.c.) carrier from $X$ to the set of all nonempty closed subsets of $E^{1}$.

It is well known and easily proven that a real-valued u.s.c. (1.s.c.) function on a compact space attains its maximum (minimum). However, this property does not characterize the compactness of the domain space. For example, it is easily shown that each u.s.c. (1.s.c.) function on $\Omega$, the first uncountable ordinal, attains its maximum (minimum). ${ }^{1}$ The purpose of this paper is to characterize various kinds of "compactness" in terms of u.s.c. (1.s.c.) carriers "attaining their maxima (minima)." We say that a carrier $\Phi$ attains a maximum (minimum) if the family $\{\Phi(x): x \in X\}$ has a maximal (minimal) member with respect to set inclusion.

In the sequel $X$ and $Y$ are always $T_{1}$ topological spaces, and $2^{Y}$ is the set of all nonempty closed subsets of $Y$. If $\alpha$ is any infinite cardinal, then we say that $X$ is $\alpha$-compact if each open cover of $X$ having cardinality $\leqq \alpha$ admits a finite subcover. And we say that a net is an $\alpha$-net if its domain is $\alpha$, where $\alpha$ may be any ordinal. Then we obtain the following well-known (cf. Chittenden [1]) and easily proved lemmas:

Lemma 1. $X$ is $\alpha$-compact if and only if each $\beta$-net in $X$ has a cluster point where $\beta$ is any ordinal $\leqq \alpha$.

LemMa 2. A space $X$ is compact if and only if each $\alpha$-net in $X$ has a cluster point in $X$, where $\alpha$ is any ordinal.

Received by the editors March 23, 1962 and, in revised form, August 18, 1962.

${ }^{1}$ We consider ordinals and cardinals as defined, for example, in the appendix of Kelley [3], in which an ordinal is equal to the set of its predecessors and a cardinal is an ordinal which is not equivalent to any of its predecessors. In fact, our topological terminology, unless otherwise specified is consistent with that used in Kelley [3]. 
Given an infinite cardinal $\alpha$ we say that a space $Y$ is $\alpha$-separable if $Y$ has a dense subset of cardinality $\leqq \alpha$; and is hereditarily $\alpha$-separable if each subset of $Y$ is $\alpha$-separable. (If $\omega$ is the first infinite cardinal, then " $\omega$-separable" is the same as "separable.") Now we obtain our main result.

TheORem 1. A space $X$ is $\alpha$-compact if and only if each u.s.c. (l.s.c.) carrier from $X$ to $2^{Y}$, where $Y$ is any hereditarily $\alpha$-separable space, attains a maximum (minimum).

Proof. We will prove the theorem only for the u.s.c. case; the 1.s.c. case is similar.

Suppose $\Phi$ is an u.s.c. carrier from an $\alpha$-compact space $X$ to $2^{Y}$, where $Y$ is some hereditarily $\alpha$-separable space. Let $Q=\{\Phi(x): x \in X\}$ and let $\mathfrak{e}=\left\{\Phi\left(x_{i}\right): i \in L\right\}$ be any chain in $a$. We will show, using Zorn's lemma, that there exists a $\Phi(x)$ such that Ue $\subset \Phi(x)$, which will complete the proof. Let $D$ be a dense subset of Ue which is wellordered by some cardinal $\beta \leqq \alpha$. Now pick $a_{1} \in L$ so that $\Phi\left(x_{a_{1}}\right)$ contains the first element of $D$. By transfinite induction, assume we have chosen for each $\gamma<\delta$ where $\delta<\beta$ an $a_{\gamma} \in L$ so that the set $C_{\gamma}=\mathrm{Ue}$ $-\left(\cup\left\{\Phi\left(x_{a_{\xi}}\right): \xi<\gamma\right\}\right)^{-}$is nonempty and $\Phi\left(x_{a_{\gamma}}\right)$ contains the first element of $D \cap C_{\gamma}$. Now consider $\delta$. If $C_{\delta}=\Lambda$, then we terminate the induction. If $C_{\delta} \neq \Lambda$, we choose $a_{\delta} \in L$ so that $\Phi\left(x_{a_{\delta}}\right)$ contains the first element of $D \cap C_{\delta}$. Let $A$ be the set of such $a_{\delta}$. Then clearly $A$ becomes a well-ordered subset of $L$ whose cardinality is $\leqq \beta \leqq \alpha$, for which we have $\operatorname{UeC}\left(\cup\left\{\Phi\left(x_{a}\right): a \in A\right\}\right)^{-}$. By $\alpha$-compactness, the net $\left\{\left(a, x_{a}\right): a \in A\right\}$ has a cluster point $x \in X$. Next, let $a \in A$ and $y \in \Phi\left(x_{a}\right)$. Since $Y$ is $T_{1}$ and $\Phi$ is u.s.c., the set $V=\{z: \Phi(z) \subset Y-\{y\}\}$ is open. If $y \notin \Phi(x)$, then $x \in V$ and there exists a $b \geqq a$ such that $\Phi\left(x_{b}\right) \subset Y-\{y\}$, whence $y \notin \Phi\left(x_{b}\right)$. But this contradicts the fact that $\Phi\left(x_{a}\right) \subset \Phi\left(x_{b}\right)$. Thus $y \in \Phi(x)$ and $U\left\{\Phi\left(x_{a}\right): a \in A\right\} \subset \Phi(x)$. Since $\Phi(x)$ is closed, we then have $\operatorname{Ue} \subset \Phi(x)$. (Note: in the 1.s.c. case the $T_{1}$-ness of $Y$ is not needed.)

Suppose that each u.s.c. carrier from $X$ to $2^{Y}$, where $Y$ is any hereditarily $\alpha$-separable space, attains a maximum. Let $\delta$ be the first car linal such that $X$ is not $\delta$-compact. If $\delta$ is nonexistent or if $\alpha<\delta$, then $X$ is $\alpha$-compact. So assume $\delta \leqq \alpha$. By Lemma 1 there exists a $\delta$ net $\left\{\left(\gamma, x_{\gamma}\right): \gamma<\delta\right\}$ in $X$ having no cluster point. Putting, for each $\gamma<\delta, \quad V_{\gamma}=X-\left(\left\{x_{\xi}: \xi \geqq \gamma\right\}\right)^{-}$, we obtain a family of open sets $\left\{V_{\boldsymbol{\gamma}}: \gamma<\delta\right\}$ covering $X$ so that $V_{\xi} \subset V_{\gamma}$ whenever $\xi<\gamma$ and $\cup\left\{V_{\xi}: \xi<\gamma\right\} \neq X$ for any $\gamma<\delta$. Now we define $\Phi$ on $X$ to $2^{\delta}$ by putting $\Phi(x)=\left\{\xi: \xi \leqq \gamma_{x}\right\}$ where $\gamma_{x}$ is the first ordinal $\gamma$ for which $x \in V_{\gamma}$. To show that $\Phi$ is u.s.c. we must show that $W^{\prime}=\{x: \Phi(x) \subset W\}$ is open 
in $X$ for each open set $W$ in $\delta$ ( $\delta$ has the order topology). In case $W=\delta$, we clearly have $W^{\prime}=X$. In case $W \neq \delta$, let $\gamma$ be the first member of $\delta-W$. If $\gamma=0$, then $W^{\prime}=\Lambda$. If $\gamma \neq 0$, then clearly $W^{\prime}$ $=\bigcup\left\{V_{\xi}: \xi<\gamma\right\}$, which is open in $X$. Thus, $\Phi$ is an u.s.c. carrier from $X$ to $2^{\delta}$ (where $\delta$ is $\alpha$-hereditarily separable) which obviously has no maximum. This contradicts the assumption that $\delta \leqq \alpha$, which finishes the proof.

Now from the above theorem and Lemma 2 it easily follows that

Theorem 2. A space $X$ is compact if and only if each u.s.c. (l.s.c.) carrier from $X$ to any $2^{Y}$ attains a maximum (minimum).

As a corollary of Theorem 2 we of course obtain the result that each real-valued u.s.c. (1.s.c.) function on a compact space attains its maximum (minimum). As another consequence: If $2^{Y}$ is topologized so that any continuous function $f$ from $X$ to $2^{Y}$ becomes also an u.s.c. (and/or 1.s.c.) carrier, then $f$ attains a maximum (and/or minimum) provided $X$ is compact. (E.g. if $Y$ is a bounded metric space, give $2^{Y}$ the Hausdorff metric topology. See Michael [4] for this and other possible topologies on $2^{Y}$.)

\section{BIBLIOGRAPHY}

1. E. W. Chittenden, On general topology, Trans. Amer. Math. Soc. 31 (1929), 290-321.

2. H. Hahn, Reelle Funktionen, Akademische Verlagsgesellschaft, Leipzig, 1932.

3. J. L. Kelley, General topology, Van Nostrand, New York, 1955.

4. E. A. Michael, Topologies on spaces of subsets, Trans. Amer. Math. Soc. 71 (1951), 152-182.

University of California at Santa Barbara 